\title{
Impedance Sensitivity-Based Corrective Method For Online Voltage Control in Smart Distribution Grids
}

\author{
Khaled Alzaareer ${ }^{a}, *$, Maarouf Saad ${ }^{a}$, Dalal Asber ${ }^{b}$, Serge Lefebvre ${ }^{b}$, Laurent Lenoir ${ }^{b}$ \\ a Department of electrical engineering at Quebec University (École de technologie supérieure), Montreal, QC, Canada \\ ${ }^{\mathrm{b}}$ Hydro-Quebec Research Institute, Montreal, QC, Canada \\ *Corresponding author at: Department of electrical engineering at Quebec University (École de technologie supérieure), Montreal, QC, Canada \\ Email address: khaled.alzaareer.1@ens.etsmtl.ca (Khaled Alzaareer)
}

\begin{abstract}
With the rapid increase in hosting large penetration levels of Distributed Generation (DG), voltage stability problem has raised a main concern for distribution networks. This study proposes a new centralized voltage control method following a security purpose for active distribution grids. The method is based on a sensitivity analysis to optimally dispatch the control variables. The sensitivity analysis uses a Thevenin- based load impedance margin (TLIM) derived from the nodal measurements to take into the consideration the changes in the system operation, especially those caused by the rapid-response devices of DGs. Sensitivity of load and equivalent impedances to control variables is investigated via the derivation of nodal voltage and current with respect to control variables. Accordingly, the contribution of each control variable in change TLIM and, hence, in voltage control can accurately be obtained. The changes of the impedances of pilot bus, which has the smallest value among all the TLIMs, are formulated in a multi-step optimization problem in terms of impedance sensitivities for the optimal dispatch of controls. The proposed sensitivities and their application in voltage control are successfully validated by a 11-kV distribution grid including 77 bus and hosting 22 DG units. Simulation results show the validity and the accuracy of the proposed sensitivity method in voltage control during different scenarios.
\end{abstract}

Keywords: Voltage Control; Sensitivity Analysis; Load Impedance Margin; Smart Grids; Distributed Generation; Distribution Networks. 


\section{Introduction}

Integration of a high penetration level of DG units has brought significant operational problems upon the distribution networks [1]. Over and under voltages are one of the main problems caused by the intermittent generation of DGs. Since DG units have the ability to provide ancillary services, numerous research efforts have focused to utilize DG units in voltage control. A review of the voltage control models proposed for smart distribution networks is presented in [2]. The optimization-based technique is one of these approaches to obtain the optimal output power of DG units [3]-[19].

A coordinated voltage control for active distribution networks is proposed in [3] to show the ability of DG units to regulate the system voltage. In [4], a coordinated voltage control for distribution networks is presented to maintain voltages at their set-point value. A centralized voltage control model based on sensitivity analysis is proposed in [5] to minimize the curtailment in active power production of DG units. A short-term scheduling of a distribution system is presented in [6] to minimize the variation in the output of DG units and node voltages. An optimal voltage control is developed in [7] to reduce the voltage deviation in distribution networks using DG units. The authors in [8] proposed an optimal voltage control to minimize the deviation in the voltage at pilot bus and the power production by DG units. A new mutation fuzzy adaptive particle swarm optimization algorithm is presented in [10] to mitigate the overvoltage and minimize total loss in active distribution systems. A coordinated voltage control of tap changers and DG units is presented in [13] to maximize daily DGs production and minimize the daily losses. A voltage control framework for day-ahead operation is presented in [14] with the objective to minimize the power losses and voltage violations in active distribution grids. The voltage control scheme developed in [15] aims to enable high solar penetrations in distribution systems while minimizing the voltage deviations and tap operations. In [16], advanced voltage control scheme is proposed for smart microgrids to maximize power generated by DG units. The control approach presented in [17] exploits the reactive power capability of DG units to minimize the power losses and mitigating overvoltages. In [18], the optimal power flow is used for voltage control in medium voltage networks with the objective to minimize the curtailment of DG units and reduce the shedding of controllable loads. The aforementioned methods formulate the problem as a single step optimization. To compensate the modeling inaccuracies, the authors in [19] have used a Model Predictive Control (MPC) to correct the voltages in active distribution networks. Multi-step optimization is used in the literature to speed-up the computation and to avoid numerical problems [20],[21]. It can be also used for large scale systems.

Generally speaking, the target voltage or normal operating limits of optimization-based voltage control may follow a security or economical purpose. However, all models proposed in the literature are designed for economic purposes (i.e. minimize the control cost or network losses) or voltage deviation minimization. All researchers focus only on keeping voltage magnitudes of distribution network within normal limits. However, the acceptable voltage magnitude cannot ensure that the system is stable [22]. According to [23], for a highly reactive power compensated system, voltage instability could happen even if the voltage magnitude is close to the nominal value.

The continuity in integration high penetration levels of DGs in distribution grids could impose a new challenge on network stability. This is due to displacement of a significant portion of the synchronous generation and increase the electrical distances between nodes [24]. Moreover, DG unavailability (or outage) and the continuously fast load increase can also significantly affect the voltage stability in distribution networks. Besides, some types of DG units (i.e. fixed speed wind turbines) always consume reactive power, which may cause voltage instability in distribution systems [25]. Another essential issue is that when transmission networks meet accidents, the voltage stability in distribution systems may be affected during post disturbances periods. For all these reasons, it is necessary to pay more attention for voltage control following security purposes (i.e. voltage stability issues) in future distribution grids.

Several techniques have been proposed for voltage stability analysis in distribution networks: continuation power flow CPF [26], probabilistic evaluation [27] and modal analysis technique [28]. However, all these approaches require extensive calculations that are not suitable for real-time applications. Several voltage stability indicators have been presented by reducing the distribution network into two-bus system [29]-[31]. However, those indicators are derived only at one operating point and none of them can involve the dynamic nonlinear behavior of loads.

Since power systems are highly nonlinear, the equivalent network would be adequate for voltage stability assessment under different conditions. With the development of phasor measurement units (PMUs), the situational awareness of the network operators is increased by developing new approaches for voltage stability assessment. In the meanwhile, to include the dynamic nonlinear behavior of power systems, the equivalent nodal analysis developed in [32] was extended to be used in distribution networks [33] for voltage stability assessment. The same concept was also used in [25] to detect the voltage instability of fixed-speed induction generator in distribution networks. The equivalent nodal analysis is simple such that Thevenin circuit seen by a load bus can be easily obtained and, then, incorporated 
into voltage stability assessment. Moreover, the parameters of equivalent impedances can be updated in case of the changes in the system operation, especially those caused by the rapid-response devices of DGs.

The existing Thevenin based methods have a lack of guiding information regarding the decisions of control measures. Thus, performing sensitivity analysis on the parameters of the Thevenin circuits is necessary for voltage stability prediction and control. The information of how each control variable contributes in voltage control is an important issue for network operators.

In this regard, this paper proposes a voltage control model following a security purpose by applying the optimal changes of DG production. On the basis of that, a sensitivity analysis is proposed on the terms of TLIM, which is derived from equivalent nodal analysis, to investigate the optimal dispatch of control variables. The key contributions of this study are:

- Using the common optimization methods for voltage control in distribution networks, the normal operating limits of voltage follow an economical purpose. In contrast, this work uses a voltage stability index, namely TLIM, as a target for voltage control in distribution networks. In other words, not only the voltage profile is considered as a main goal in this study, but also the voltage stability is taken into account.

- The sensitivity analysis is presented for the first time in order to evaluate the sensitivity of the load and the equivalent impedances of a load bus to control variables. These sensitivities are derived via the derivation of nodal voltage and current with respect to control variables. The proposed sensitivities provide an analysis of not only how other buses affect the impedances of a specific load bus but also how the specific bus can affect the impedances of other load buses.

- Compared with the well-known sensitivity techniques that oriented to find the contribution of each control variable in change of voltage stability margin, the proposed sensitivity approach does not require a detailed system model and complex calculation to find the critical point via CPF or the singularity of Jacobian matrix. This feature makes the proposed sensitivity method suitable for real-time voltage control in distribution networks.

- Simulation results successfully verify the proposed impedance sensitivities and validate that they can accurately find the optimal dispatch of control variables and demonstrate their accuracy in voltage control.

The rest of the paper is as follows: Section II presents the Equivalent Nodal Analysis to formulate TLIM. Section III uses the sensitivity analysis to derive the change of TLIM due to control variable changes. The optimization-based Voltage Control Scheme is presented in section IV. Section VI shows the results and discussion.

\section{Thevenin based load impedance margin TLIM}

TLIM is an index for estimating the voltage stability margin, derived from the equivalent nodal analysis and based on Thevenin's equivalent theory. In general, the nodal current equation of any power system can be written as $[\mathrm{V}]=[\mathrm{Y}][\mathrm{I}]$. Where $\mathrm{Y}$ is the network bus admittance, I and $\mathrm{V}$ are bus current and voltage vectors, respectively. The network buses can be classified into generator buses $\mathrm{G}=\{1, \ldots, \mathrm{M}\}$, load buses $\mathrm{L}=\{\mathrm{M}+1, \ldots, \mathrm{N}\}$ and Tie buses $\mathrm{T}=$ $\{\mathrm{N}+1, \ldots, \mathrm{R}\}$. By eliminating the tie buses and reorganizing the nodal equation, we can obtain:

$$
\left[\begin{array}{c}
V_{G} \\
V_{L}
\end{array}\right]=\left[\begin{array}{ll}
Z_{G G} & Z_{G L} \\
Z_{L G} & Z_{L L}
\end{array}\right]\left[\begin{array}{c}
I_{G} \\
-I_{L}
\end{array}\right]
$$

Where $Z$ is the network impedance matrix. From (1), the load voltage can be expressed as:

$$
V_{L}=Z_{L G} I_{G}-Z_{L L} I_{L}
$$

The term $Z_{L G} I_{G}$ can be written as:

and the term $Z_{L L} I_{L}$ can be written as:

$$
Z_{L G} I_{G}=\left(Z_{i 1} I_{1}+\cdots+Z_{i M} I_{M}\right)=\sum_{\mathrm{j} \epsilon G} Z_{i j} I_{j}
$$

$$
Z_{L L} I_{L}=\left(Z_{i 1} I_{M+1}+\cdots+Z_{i i} I_{i}+\cdots+Z_{i N} I_{N}\right)=\left(Z_{i i}+\sum_{\substack{\mathrm{j} \epsilon L \\ i \neq j}} Z_{i j} \frac{I_{j}}{I_{i}}\right) I_{i}
$$

Thus, the voltage at load bus i $\epsilon L$, can be obtained as

$$
V_{i}=\sum_{\mathrm{j} \epsilon G} Z_{i j} I_{j}-\left(Z_{i i}+\sum_{\substack{\mathrm{j} \epsilon L \\ i \neq j}} Z_{i j} \frac{I_{j}}{I_{i}}\right) I_{i}
$$


where $I_{i}$ and $I_{j}$ are the current injected at load buses i and j respectively; $Z_{i i}$ and $Z_{i j}$ are the self and coupling impedance of bus i respectively and can be directly obtained using the submatrix $Z_{L L}$

Since (5) the can be written in terms of the Thevenin parameters as $V_{i}=V_{e q, i}-Z_{e q, i} I_{i}$, we conclude that:

$$
Z_{e q, i}=Z_{i i}+\sum_{\substack{\mathbf{j} \epsilon L \\ i \neq j}} Z_{i j} \frac{I_{j}}{I_{i}}
$$

Where $V_{e q, i}$ and $Z_{e q, i}$ are the equivalent voltage and impedance referred to load bus i respectively. Based on the Thevenin equivalent theory, the maximum power transfer to a load bus occurs when the load impedance $\left|Z_{L}\right|$ matches the equivalent impedance of the rest of the system $\left|Z_{e q}\right|$. TLIM at any load bus i can then be obtained as:

$$
\operatorname{TLIM}_{i}=\frac{\left|z_{L, i}\right|-\left|z_{e q, i}\right|}{\left|z_{L, i}\right|} \quad \mathrm{i} \in L
$$

Where $Z_{L, i}$ is the load impedance of bus $i$ and can be found as:

$$
Z_{L, i}=\frac{V_{i}}{I_{i}}
$$

Where $V_{i}$ and $I_{i}$ are the measured voltage and current at bus i respectively. Based on (6)-(8), it is clear that the load and the equivalent impedances are based on the nodal current and voltage measurements and the network impedance matrix. This means that this approach requires PMUs to be installed at the load buses to measure the nodal voltage and current. The nodal measurements and the data of Y matrix are sent through Supervisory Control and Data Acquisition (SCADA) system onward to control center. The calculations are then done in control center to obtain the optimal changes in control variables. Those changes are sent to update the reference set-points of controls.

Remark: For practical systems, appropriate PMU placement techniques can be performed first while ensuring the full observability of the network. PMUs can be installed at critical buses where the power is supplied to a couple of nodes or feeders. Thus, all the downstream nodes of each critical bus can be seen as one lumped load impedance. This issue is not part of the present paper and will be studied in our future works.

The value of the TLIM ranges from 0 to 1 , and TLIM $=0$ represents the instability point where $\left|Z_{e q}\right|=\left|Z_{L}\right|$ (as proposed in other Thevenin- based indices [25],[34],[35]). For large power systems, the smallest value among all the TLIMs can be selected to represent the system margin (i.e. pilot node) as [32]:

$$
\operatorname{TLIM}=\min \left(\operatorname{TLIM}_{i}\right) \quad \mathrm{i} \epsilon L
$$

To show the behavior of the proposed TLIM in determining the distance to voltage instability, let us consider the $\mathrm{R}-\mathrm{X}$ diagram in Fig. 1. If the load impedance $\left|Z_{L}\right|$ is moved from $\mathrm{B}$ to $\mathrm{B}^{\prime}$ or the radius of the equivalent impedance $\left|Z_{e q}\right|$ is increased from A to $\mathrm{A}^{\prime}$, the TLIM will decrease. If $\left|Z_{L}\right|$ is located inside the circle with a radius $\left|Z_{e q}\right|$, the system is unstable. Therefore, it is necessary to keep $\left|Z_{L}\right|$ far away from the circle of $Z_{e q}$ to maintain a safe stability and operation.

It is worth mentioning that $\left|Z_{e q}\right|$ is not a constant value and varies following the dynamic behavior of power systems. Since the derived TLIM is based on equivalent nodal analysis, it can easily capture any change in system operation and, hence, the increment or decrement in $\left|Z_{\text {eq }}\right|$ as illustrated in (6).

\section{TLIM-based sensitivity analysis}

According to section II, any change in load power consumption (i.e. load current) or voltage of load bus i will result in an increase or decrease in the load impedance. Similarly, any change in the current or voltage of other load buses will result in an increment or decrement in the equivalent impedance seen by the load bus i. To explain how control variables can vary the impedances referred to a load bus, let us consider the small distribution system shown in Fig. 2 . The network consists of 3 load buses (1, $2 \& 4$ ) and one bus for DG installation (bus 3). It is assumed that DG unit operates with constant output power. As we explained in section II, to assess the voltage stability at bus 4, the network can be simplified to Thevenin equivalent circuit as shown in Fig.2a. Any increase or decrease in power injected by DG 
unit will cause the voltages and branch currents to change by $\Delta V_{L}, \Delta I_{L}$ respectively. By referring to (6) \& (8), we can conclude that the load and the equivalent impedances referred to a load bus 4 will vary by $\Delta Z_{L}, \Delta Z_{\text {eq }}$ respectively, as shown in Fig.2b. Accordingly, the voltage stability of any load bus can be estimated as expressed in (7).

However, no further information is available to guide the decisions of control measures. To provide such information, sensitivity analyses on the load and the equivalent impedances is performed in this study to examine the impact of control variables (i.e. nodal power injection) on these impedances. Accordingly, the contribution of each control variable in change TLIM can be easily obtained. This helps network operators to take a global measure to improve the nodes voltages as well as the voltage stability.

\subsection{Sensitivity of load bus voltages to control variables}

Sensitivity of a load bus voltage to any change in control variable $u_{x}$ can be calculated as:

$$
\frac{d V_{i}}{d u_{x}}=e^{j \theta_{i}}\left(\frac{d\left|V_{i}\right|}{d u_{x}}+j\left|V_{i}\right| \frac{d \theta_{i}}{d u_{x}}\right)
$$

Where $\left|V_{i}\right|$ and $\theta_{i}$ are the node $\mathrm{i}$ voltage magnitude and angle respectively. Assuming that the control variables are only the power injected by DG, $d\left|V_{i}\right| / d u_{x}$ and $d \theta_{i} / d u_{x}$ can be obtained from the inverse of Jacobian matrix $J$ as:

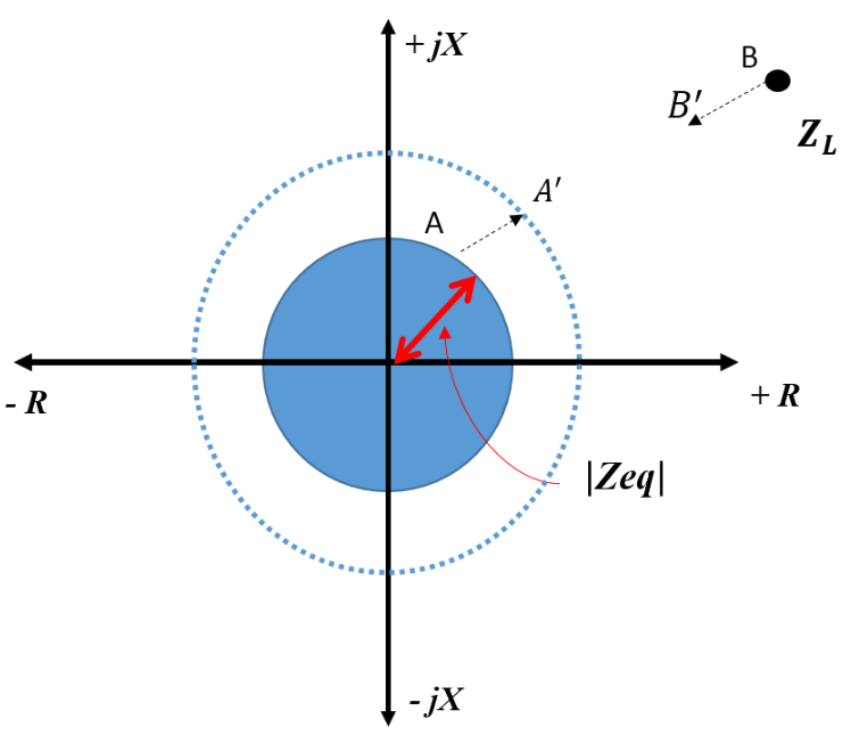

Fig.1: $\mathrm{R}-\mathrm{X}$ diagram for TLIM

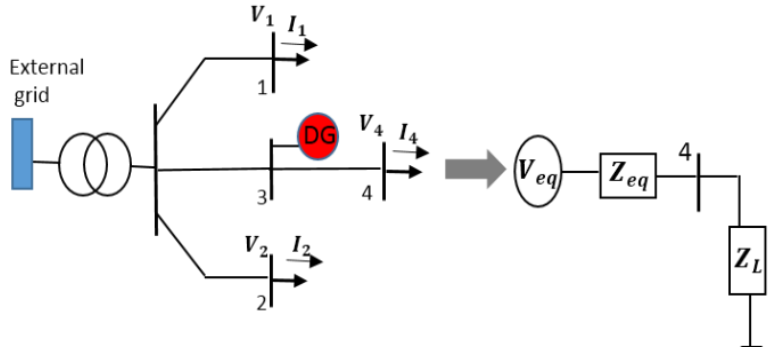

(a)

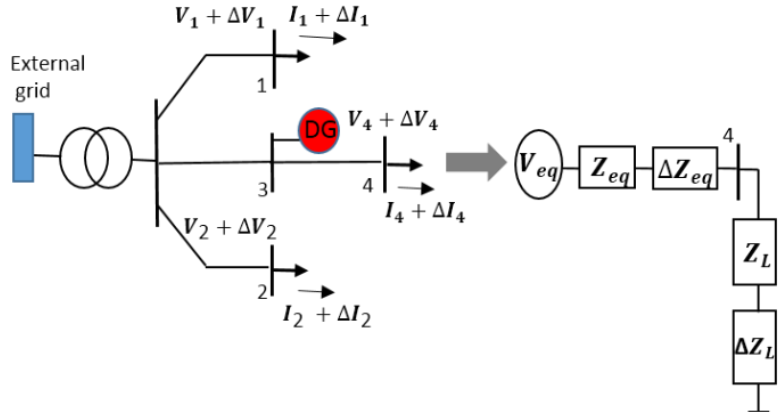

(b)

Fig. 2. Simple distribution system with simplified circuit referred to bus 4 (a) before and (b) after injecting power by DG. 


$$
\left[\begin{array}{c}
\Delta|V| \\
\Delta \theta
\end{array}\right]=[J]^{-1}\left[\begin{array}{c}
\Delta P \\
\Delta Q
\end{array}\right]=\left[\begin{array}{ll}
\frac{\partial|V|}{\partial P} & \frac{\partial|V|}{\partial Q} \\
\frac{\partial \theta}{\partial P} & \frac{\partial \theta}{\partial Q}
\end{array}\right]\left[\begin{array}{c}
\Delta P \\
\Delta Q
\end{array}\right]
$$

Where $\partial|V| / \partial P$ and $\partial|V| / \partial Q$ are the sensitivity vectors of nodal voltage magnitude to real and reactive power injection, respectively. $\partial \theta / \partial P$ and $\partial \theta / \partial Q$ are the sensitivity vectors of nodal voltage angle to real and reactive power injection, respectively.

\subsection{Sensitivity of load currents to control variables}

The load current at any bus i can be calculated as:

$$
I_{i}=\frac{S_{i}^{*}}{V_{i}^{*}}
$$

Where $S_{i}$ is complex power of bus i. Thus, the sensitivity of any load current to control variable can be obtained by:

$$
\frac{d I_{i}}{d u_{x}}=\frac{d\left\{\frac{S_{i}^{*}}{V_{i}^{*}}\right\}}{d u_{x}}=\frac{V_{i}^{*} \frac{d S_{i}^{*}}{d u_{x}}-S_{i}^{*} \frac{d V_{i}^{*}}{d u_{x}}}{V_{i}^{* 2}}
$$

At constant load impedance, the term $S_{i}{ }^{*}$ can be written as:

$$
S_{i}^{*}=\frac{\left|V_{i}\right|^{2}}{Z_{L, i}}
$$

Thus, the sensitivity $S_{i}{ }^{*}$ to control variable can be calculated as:

$$
\frac{d S_{i}^{*}}{d u_{x}}=\frac{2 v_{i}}{Z_{L, i}} \frac{d V_{i}}{d u_{x}}
$$

By substituting (14) \& (15) into (13), we obtain:

$$
\frac{d I_{i}}{d u_{x}}=\frac{I_{i}}{V_{i}{ }^{*}}\left(2 \frac{d V_{i}}{d u_{x}}-\frac{d V_{i}^{*}}{d u_{x}}\right)
$$

\subsection{Sensitivity of load impedances to control variables}

The sensitivity of load impedance $Z_{L, i}$ to control variable $\mathrm{u}_{x}$ can be found by taking the derivation of (8) with respect to $\mathrm{u}_{x}$ :

$$
\frac{d Z_{L, i}}{d u_{x}}=\frac{d\left\{\frac{V_{i}}{I_{i}}\right\}}{d u_{x}}=\frac{I_{i} \frac{d V_{i}}{d u_{x}}-V_{i} \frac{d I_{i}}{d u_{x}}}{I_{i}{ }^{2}}
$$

By substituting (16) into (17), we obtain:

$$
\frac{d Z_{L, i}}{d u_{x}}=\frac{\left(1-\frac{2 V_{i}}{V_{i}^{*}}\right) \frac{d V_{i}}{d u_{x}}+\frac{V_{i}}{V_{i}^{*}} \frac{d V_{i}^{*}}{d u_{x}}}{I_{i}}
$$

\subsection{Sensitivity of equivalent impedances to control variables}

The sensitivity of the equivalent impedance referred to a load bus i to control variable $u_{x}$ can be calculated by taking the derivation of (6) with respect to $u_{x}$ as:

$$
\frac{d Z_{e q, i}}{d u_{x}}=\sum_{\substack{\mathbf{j} \epsilon L \\ i \neq j}} Z_{i j} \frac{d\left(\frac{I_{j}}{I_{i}}\right)}{d u_{x}}=\sum_{\substack{\mathbf{j} \epsilon L \\ i \neq j}} Z_{i j} \frac{I_{i} \frac{d I_{j}}{d u_{x}}-I_{j} \frac{d I_{i}}{d u_{x}}}{I_{i}{ }^{2}}
$$

By substituting (16) for the buses i \& j into (19), we obtain: 


$$
\frac{d Z_{e q, i}}{d u_{x}}=\sum_{\substack{j=1 \\ i \neq j}} \frac{Z_{i j} I_{j}}{I_{i}}\left(\frac{2}{V_{j}^{*}} \frac{d V_{j}}{d u_{x}}-\frac{2}{V_{i}^{*}} \frac{d V_{i}}{d u_{x}}-\frac{1}{V_{j}^{*}} \frac{d V_{j}^{*}}{d u_{x}}+\frac{1}{V_{i}^{*}} \frac{d V_{i}^{*}}{d u_{x}}\right)
$$

\subsection{Calculation the change of TLIM}

By obtaining the sensitivities $d Z_{L, i} / d u_{x}$ and $d Z_{e q, i} / d u_{x}$, we can obtain that:

$$
\begin{aligned}
\Delta Z_{L, i} & =\sum_{x=1}^{M} \frac{d Z_{L, i}}{d u_{x}} \Delta u_{x} \\
\Delta Z_{e q, i} & =\sum_{x=1}^{M} \frac{d Z_{e q, i}}{d u_{x}} \Delta u_{x}
\end{aligned}
$$

Whrere $\Delta Z_{L, i} \& \Delta Z_{e q, i}$ are the change in the load and equivalent impedances referred to load bus i respectively. $\Delta u_{x}$ represents the change in the control variable x. $M$ represents the number of control variables. The new TLIM that results due to the changing in control variables can be found as:

$$
T L I M_{i, \text { new }}=\frac{\left|Z_{L, i}+\Delta Z_{L, i}\right|-\left|z_{e q, i}+\Delta Z_{e q, i}\right|}{\left|z_{L, i}+\Delta Z_{L, i}\right|}
$$

From (21)-(23), it is clear that the change in any control variable (i.e. the power injected) at a particular load bus will not only affect the voltage stability (TLIM) of its own bus, but also the stability of other buses. According to (18) $\&$ (20), the proposed sensitivities can be used to investigate the coupling effect of the load buses in TLIM and to assess the impact of change of the power injected by any DG to the load and the equivalent impedances and, hence, voltage stability.

\section{Voltage control scheme}

The voltage control approach proposed in this study is based on a multi-step optimization to smoothly correct the voltages. The approach is inspired from MPC to predict the behavior of the system over interval of $n$ discrete steps [19]. Since the pilot bus can determine the overall performance of the network, the main objective in this study is to minimize the deviation in the margin (TLIM) of the pilot bus. Accordingly, a standard Quadratic Programming problem can be formulated as:

$$
\min \sum_{k=0}^{n-1}\left\|\operatorname{Ref}-\operatorname{TLIM}_{p}(t+k)\right\|^{2}
$$

Ref is the target value for the index TLIM and equals $1 . \mathrm{t}$ represents the time instant and $\mathrm{n}$ represents the length of the prediction or control horizon. $\operatorname{TLIM}_{p}(t+k)$ is the predicted voltage stability margin of pilot bus given the measurements at time instant t. By substituting (23) into (24), (24) becomes:

$$
\min \sum_{\boldsymbol{d}_{k=0}}^{n-1}\left\|\frac{\left|z_{e q, p}+\Delta Z_{e q, p}(t+k)\right|}{\left|Z_{L, p}+\Delta Z_{L, p}(t+k)\right|}\right\|^{2}
$$

Where $\Delta Z_{L, p}(t+k)$ and $\Delta Z_{\text {eq,p }}(t+k)$ represent the change of the load and the equivalent impedances referred to pilot bus at time $t+k$ respectively. The subletter p denotes for pilot bus. By substituting (21) \& (22) into (25), we obtain: 


$$
\min \sum_{k=0}^{n-1} \| \frac{\left|Z_{e q, p}+\sum_{x=1}^{M} \frac{d Z_{e q, p}}{d u_{x}} \Delta u_{x}(t+k)\right|}{\left|Z_{L, p}+\sum_{x=1}^{M} \frac{d Z_{L, p}}{d u_{x}} \Delta u_{x}(t+k)\right|}||^{2}
$$

Where $\Delta u_{x}(t+k)$ is the change of control variable $\mathrm{x}$ at time $t+k$. For practical purposes, another objective function can be included to minimize the changes of the control variables. As a result, the following optimization problem can be used:

$$
\min w \sum_{k=0}^{n-1}\left\|\frac{\left|z_{e q, p}+\frac{d z_{e q, p}}{d u} \Delta u(t+k)\right|}{\left|z_{L, p}+\frac{d z_{L, p}}{d u} \Delta u(t+k)\right|}\right\|_{G}^{2}+(1-w) \sum_{k=0}^{n-1}\|\Delta u(t+k)\|_{G}^{2}+\|\varepsilon\|_{H}^{2}
$$

Subject to:

$$
\begin{gathered}
-\varepsilon_{1} A+V_{i}^{\text {min }} \leq V_{i}(t+k) \leq V_{i}^{\text {max }}+\varepsilon_{2} A \\
V_{i}(t+k)=V_{i}(t+k-1)+\frac{\partial|V|}{\partial u} \Delta u_{i}(t+k) \\
\Delta Q_{x}{ }^{\text {min }} \leq \Delta Q_{x}(t+k) \leq \Delta Q_{x}{ }^{\text {max }} \\
\Delta P_{x}{ }^{\text {min }} \leq \Delta P_{x}(t+k) \leq \Delta P_{x}^{\text {max }} \\
P_{x}^{\text {min }} \leq P_{x}(t+k) \leq P_{x}^{\text {max }} \\
Q_{x}{ }^{\text {min }} \leq Q_{x}(t+k) \leq Q_{x}^{\text {max }}
\end{gathered}
$$

Where $0 \leq \mathrm{w} \leq 1$ is to penalize each objective function. $\frac{d Z_{L, p}}{d u}=\left[\frac{d Z_{L, p}}{d P}, \frac{d Z_{L, p}}{d Q}\right]$ and $\frac{d Z_{e q, p}}{d u}=\left[\frac{d Z_{e q, p}}{d P}, \frac{d Z_{e q, p}}{d Q}\right]$ are the sensitivity vectors of the load and equivalent impedances of pilot bus to control variables respectively. $\frac{d Z_{L, p}}{d P}, \frac{d Z_{e q, p}}{d P}$ and $\frac{d Z_{L, p}}{d Q}, \frac{d Z_{e q, p}}{d Q}$ represent the sensitivity vectors of the load and equivalent impedances of pilot bus to real and reactive power injected by DGs respectively. $\Delta u=[\Delta P, \Delta Q]^{T}$ represents the vector of changes of control variables. ' $T$ ' represents array transposition. $\Delta P \& \Delta Q$ represents the vector of changes of real and reactive power injected by DGs respectively. $\varepsilon=\left[\varepsilon_{1}, \varepsilon_{2}\right]^{\mathrm{T}}$ is the vector of slack variables used and relax the voltage constraints. 'A' denotes a unitary vector. $G$ is a weight matrix used to penalize the "expensive" generation control variables. The weight matrix $H$ is used to penalize the slack variables. $V_{i}(t+k)$ is the predicted voltage magnitude of bus i given the measurements at time instant t. $V_{i}(t+k-1)$ is the measured voltage magnitude of bus i at time instant t. $\frac{\partial|V|}{\partial u}=\left[\frac{d|V|}{d P}, \frac{d|V|}{d Q}\right]$ is the sensitivity matrix of bus voltage magnitudes with respect to the control variables. $P_{x} \& Q_{x}$ are the real and reactive power injected by DG ' $\mathrm{x}$ ' respectively. $\Delta P_{x} \& \Delta Q_{x}$ are the change in real and reactive power injected by DG ' $\mathrm{x}$ ' respectively. $\Delta P_{x}{ }^{\min }, \Delta P_{x}{ }^{\max }, \Delta Q_{x}{ }^{\min }$ and $\Delta Q_{x}{ }^{\max }$ represent the limits for the change in real and reactive power injected by DG ' $x$ '. DG limits are included in the optimization problem (i.e. the third and fourth constraints) to protect the equipments (i.e. machines or inverters) against fast ramping and to create a cautious environment for distributing the control actions. However, those limits can be provided for use in the constraint set according to data provided by equipment's manufactures.

Remark: Several algorithms can be used to solve the optimization problem. They are very known methods for researchers. However, since the objective of this work is not to show how optimization problems can be solved, any optimization modeling tool can be used to obtain the optimal changes in control variables.

It is worth mentioning that the proposed method is more flexible such that it can incorporate conventional voltage control devices in the optimization problem to avoid any interaction between control variables. This requires the following changes in the optimization problem: 
- modify the vector of changes of control variables $(\Delta u)$ in equation (27) to contain those other devices (i.e. $\Delta u=\left[\Delta P, \Delta Q, \triangle V C D_{1}, \triangle V C D_{2}, \ldots \Delta V C D_{i}\right]^{T}$ ) where $\triangle V C D_{i}$ represent the change in the $\mathrm{i}^{\text {th }}$ other voltage control device (i.e. the voltage set-point of voltage regulators).

- add new constraints to the optimization problem such as the limits of voltage control devices and capacitor banks integer positions.

- As expressed in equations (18) and (20), the sensitivities $\frac{d Z_{e q, p}}{d u}$ and $\frac{d z_{L, p}}{d u}$ depend on the sensitivity $\frac{d V}{d u_{x}}$. As shown in section 3.1, if the control variables are only the power injected by DG, the term $\frac{d V}{d u_{x}}$ can be directly obtained from the inverse of Jacobian matrix $J$. However, for other control variables, the term $\frac{d V}{d u_{x}}$ can be found by linearizing the power flow equations $F\left(V, u_{x}\right)=0$ as (assuming that the load parameter is constant at current operating point):

$$
\frac{d F}{d V} \Delta V+\frac{d F}{d u_{x}} \Delta u_{x}=0
$$

Where $\frac{d F}{d V}$ equals to Jacobian matrix at the base case and $\frac{d F}{d u_{x}}$ is known. Thus, $\frac{\Delta V_{i}}{\Delta u_{x}}\left(\right.$ or $\left.\frac{d V}{d u_{x}}\right)$ can be easily found.

The proposed method is also flexible such that it can incorporate remotely controlled switches in the optimization problem. This also requires to modify the vector of changes of control variables $(\Delta u)$ in equation (27) to contains remotely controlled switches and to add new constraints to the optimization problem such as the connection status of remotely controlled switches. If the control variable is a remotely controlled switch, the term $\frac{d V}{d u_{x}}$ can be approximated by computing the variations of the bus voltages due to remotely controlled switch action. This information can be easily extracted from the solution of two power flow runs with a remotely controlled switch action.

The proposed algorithm can be implemented in the control center of distribution network as follows:

Step 1: the data of network admittance matrix $\mathrm{Y}$ and the nodal measurements are collected from SCADA system and PMUs.

$\underline{\text { Step 2: }}$ If the network is subjected to structural changes, update the admittance matrix Y. Otherwise, use the previous matrix.

Step 3: If the network is subjected to DG unavailability, recalculate the submatrix $Z_{L L}$. Otherwise, use the previous $\mathrm{Z}_{\mathrm{LL}}$.

Step 4: Calculate the load and the equivalent impedances and TLIM for the interested load buses using (6), (7) \& (8), and determine the pilot bus.

Step 5: Calculate the voltage and current sensitivities of pilot bus to control variables using (10) \& (16).

$\underline{\text { Step 6: }}$ : Calculate the sensitivities of the load and equivalent impedances of pilot bus to control variables using (18) \& (20).

Step 7: Construct and solve the optimization problem in (27) and apply the control actions.

If all voltages are within the normal range, process is finished. Otherwise, go to step 4.

\section{Simulation results}

To verify the applicability and robustness of the proposed method, 77-bus, $11 \mathrm{kV}$ distribution grid shown in Fig. 3 is used as a test system. The network consists of 22DG units and 53 load bus. The system base is $100 \mathrm{MVA}$. The line parameters and load data are available in [36]. The system and the proposed approach have been simulated in MATLAB under different operating conditions. LINGO software was interfaced with MATLAB to solve the real time optimization problem. 


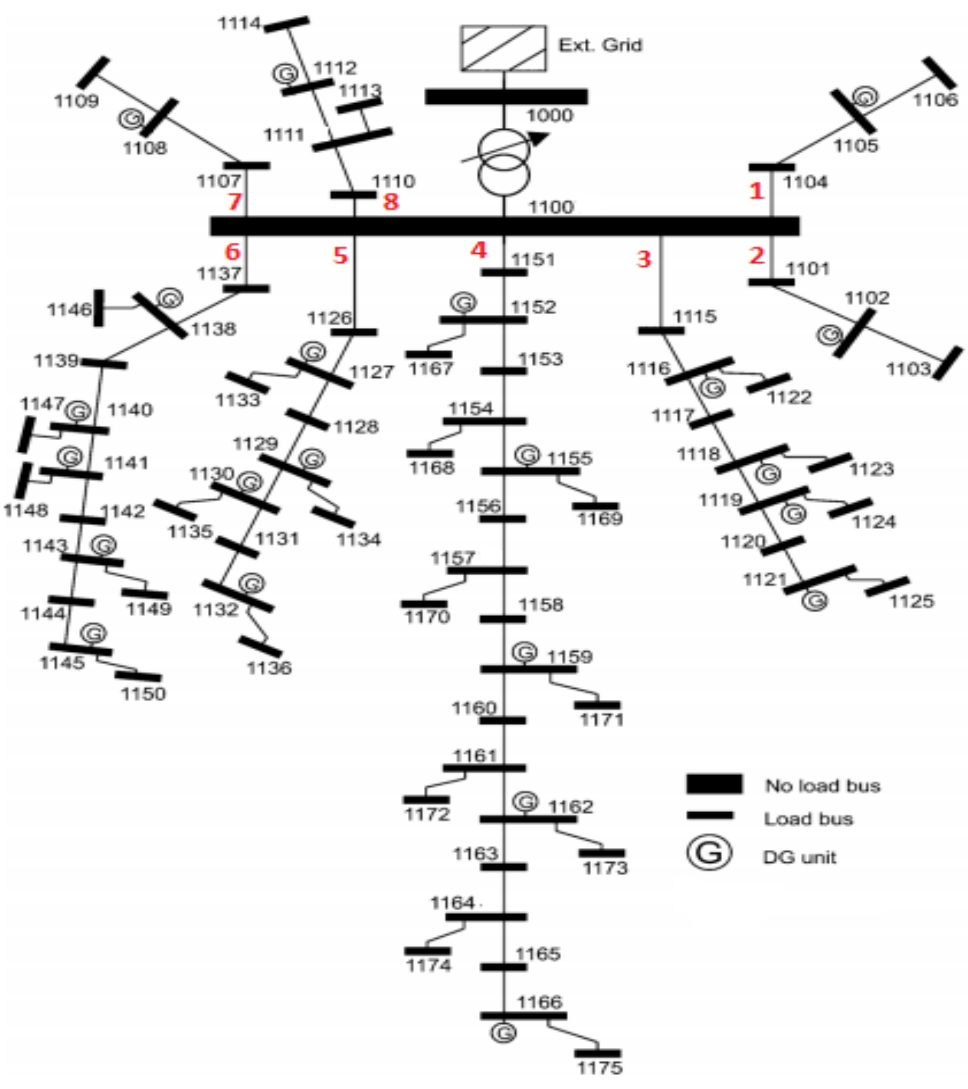

Fig.3: Topology of the test system [19]

\subsection{Verification of the impedance- based sensitivities}

In this scenario, the system is operated under base load condition with no DG integrated to the system. The sensitivities $\left|d Z_{L} / d P\right|,\left|d Z_{L} / d Q\right|$ and $\left|d Z_{\text {eq }} / d P\right|,\left|d Z_{e q} / d Q\right|$ of the load buses are demonstrated in Figs. 4 and 5 respectively. It is clear from Fig. 4 that all the sensitivities are positive, which indicates that increase in nodal active or reactive power injection will increase the load impedances. In contrast, the positive values of sensitivities in Fig. 5 indicate that increase in nodal active or reactive power injection will decrease the equivalent impedances. The resulted matrices also show that the diagonal elements are dominant which means that the change of power injection at a specific bus has a higher impact on its own $Z_{L}$ and $Z_{e q}$ than the change power at other buses. This due to fact that the power flow across the network reduces impact of power injection at a specific bus on other buses while the change at its own bus cast such impact without any losses across the network. It is also noticed that the sensitivity of the load and equivalent impedances of a particular bus to power injection at other buses in the same feeder are higher than the sensitivity due to power injection at the buses of other feeders. For example, the sensitivity of $Z_{L}$ and $Z_{e q}$ of bus 1175 to power injection at any bus in feeder 4 are higher than the sensitivity due to power injection in other feeders. The modest sensitivity in the region near the bus 1103 and bus 1175 can be demonstrated by noticing that the nodes near the bus 1103 are located at nodes far away from the bus 1175 and on other feeders.

It is worth mentioning that the sensitivities of $Z_{e q}$ of the buses located at the end of feeders 4 and 6 are significantly greater than those of the buses located at the beginning of the feeders or at the other feeders as shown in Fig.5. This is due to fact that injecting power at the buses of the end of the feeders 4 or 6 has a higher impact on other buses than injecting the power at the beginning of the feeder or the other feeders. The high difference between the sensitivities can be explained from (20) which shows that the equivalent impedance sensitivity depends on the sum of the coupling effects between buses.

From (18) and (20), we can find that the proposed sensitivities are closely related to the sensitivity terms of $[\mathrm{J}]^{-1}$. The numerical values of the sensitivities illustrate the strong relation to the values of $[\mathrm{J}]^{-1}$. For example, the bus 1175 self-sensitivity term of $[\mathrm{J}]^{-1}$ is higher than its cross-sensitivity terms. Moreover, the bus 1175 largest cross-sensitivity 
term is obtained with bus 1165 which is the nearest location to bus 1175 . This justifies the higher sensitivities of the impedances of bus 1175 to power injection at its own location and the nearest buses over other locations.

From the results, it is noticed that changing the impedances is not a local problem in which $Z_{L}$ and $Z_{e q}$ of a load bus are changed due to a disturbance (i.e. change in power injection) in its own bus or other buses network. Verification of the sensitivities of $Z_{L}$ and $Z_{e q}$ of bus 1175 to active and reactive power injection at its own bus and bus 1165 demonstrates the validity of the proposed sensitivities.

\subsection{Impedance sensitivity - based voltage control}

Application of the equivalent nodal analysis (similar to TLIM ) in voltage stability assessment has been studied in [15],[24]. Thus, this work focusses only on examination of the applicability and accuracy of the proposed sensitivities $\left|d Z_{L} / d u\right|$ and $\left|d Z_{e q} / d u\right|$ in voltage control. Application of the proposed method for a weak system and during contingency cases will be studied in the next future work.

In this test, it is assumed that the rating for DG units are 3.5-MVA with $2.6 \mathrm{MW}$ of maximum capacity. For each step of corrective actions, DG units are allowed to change their active and reactive power not more than $0.3 \mathrm{MW}$ and 0.3 MVAr respectively. The cost of using the active power and slack values are higher than the cost of using the reactive power by 10 and 800 times respectively. The normal voltage limits are considered to be within [0.98, 1.04] p.u. The controller uses only the reactive power for voltage control while the active power is only used when any voltage reaches the emergency upper limit $(=1.06 \mathrm{p} . \mathrm{u}$ in this study). It is also assumed that the controller updates the set points of control variables every 10 seconds and collects the measurements 8 seconds after updating the set points.

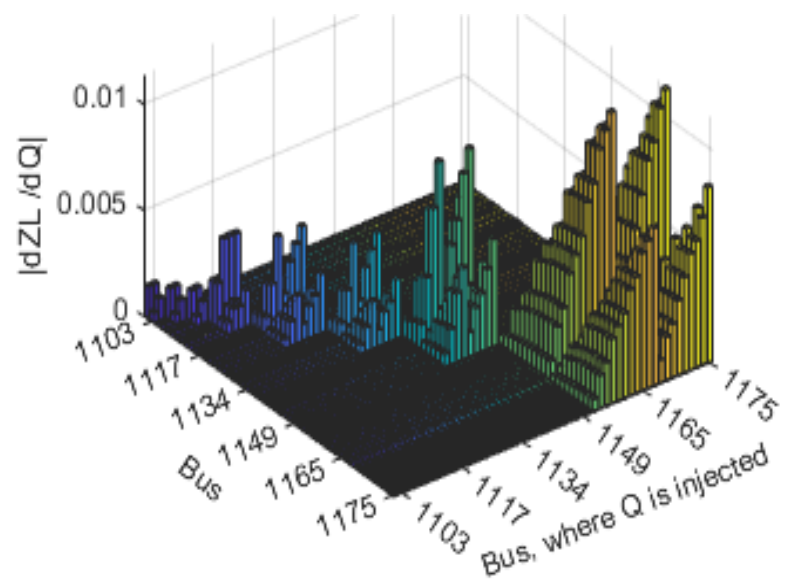

(a)

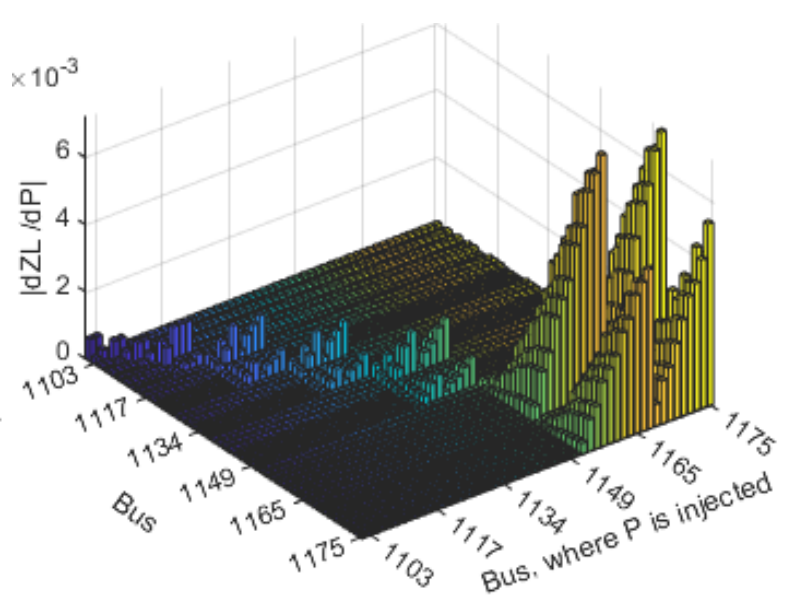

(b)

Fig.4. (a) $\left|Z_{L} / d P\right|$ and (b) $\left|Z_{L} / d Q\right|$ sensitivity matrices for base condition.

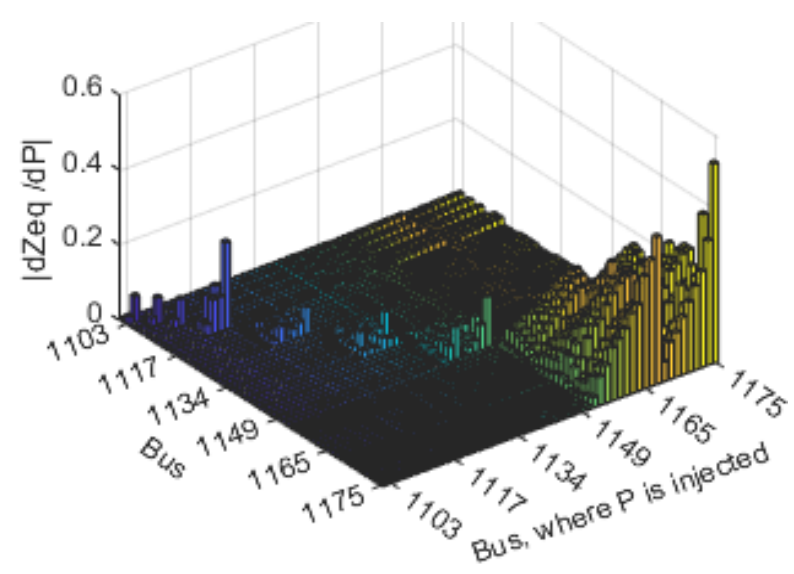

(a)

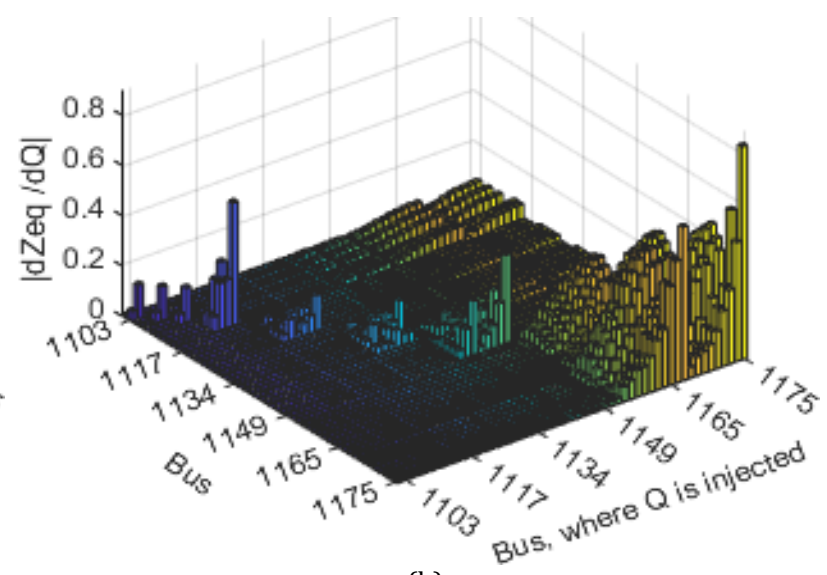

(b)

Fig.5. (a) $\left|Z_{e q} / d P\right|$ and (b) $\left|Z_{e q} / d Q\right|$ sensitivity matrices for base condition. 


\subsubsection{Under voltages scenario}

In this case, the network operates at maximum power demand so that some buses suffer from low voltage. Since all voltages are below the emergency upper limit, the reactive power outputs of DG units are only used for voltage correction. Fig.6a shows the voltages regulation starting at $\mathrm{t}=40 \mathrm{~s}$ and changing every $10 \mathrm{~s}$ by adjusting the set point of DG units. The measured voltages, currents and the sensitivities are also updated every 10s to achieve the correct voltage control. The curves in Fig.6b correspond to the most representative voltages of both DG and load buses. It is clear that the controller can improve the voltages gradually until the latter are brought back inside the normal limits. Once the voltages are restored inside the normal limits, the controller does not request further increase in reactive power. This condition is met at 70s. It is also shown from Fig.6b that although most of the DG units provide reactive power, DG located at bus 1105 was requested to still operate at the lower amount of reactive power. This is because the voltages near bus 1105 (i.e. v1105) would violate the upper limit of 1.04 p.u. It is also noticed that DG located at 1166 provide the higher amount of reactive power because v1166 is the most problematic voltage.

When all under voltages are corrected, the total compensated amount of reactive power is given in Fig. 7. It is clear that not all the DG units are used for voltage support. Some units still operate at lower amount of reactive power while others absorb reactive power.

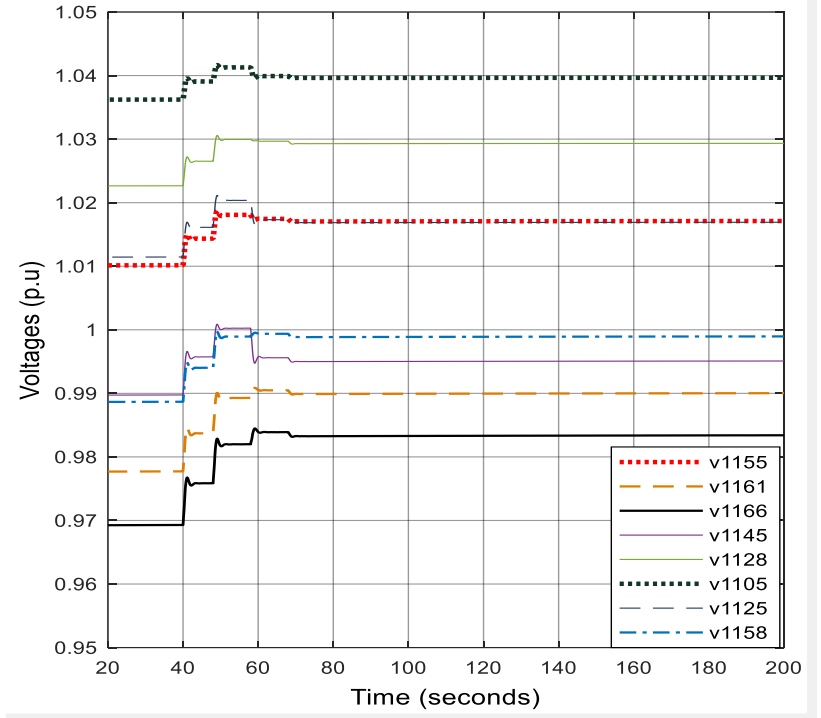

(a)

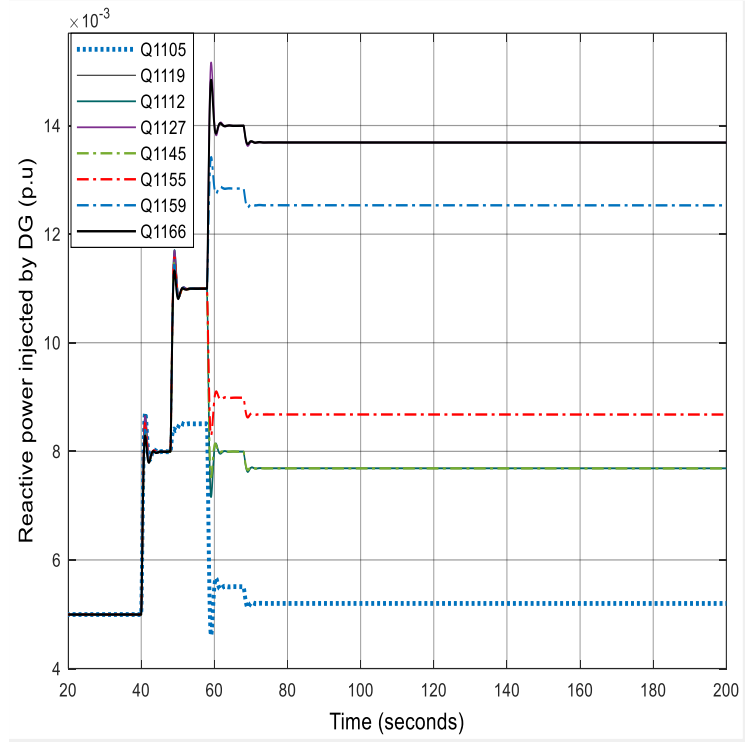

(b)

Fig.6. (a) Bus voltages \& (b) Reactive power output of the DGs for scenario 1

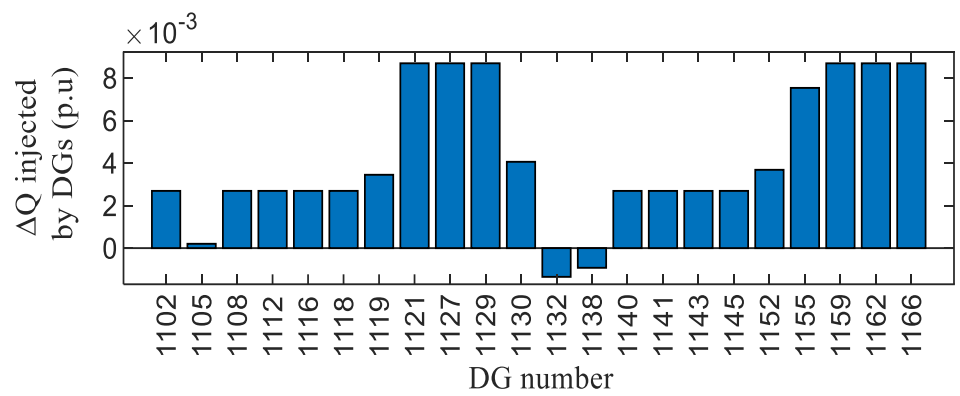

Fig.7. The total compensated reactive power outputs by DGs for scenario 1.

The node voltage profiles of two cases, namely, Vuncontrolled and Vcontrolled, are shown in Fig. 8. It is shown that the proposed approach can correct the voltages by generating 9.57MVar reactive power in total. 


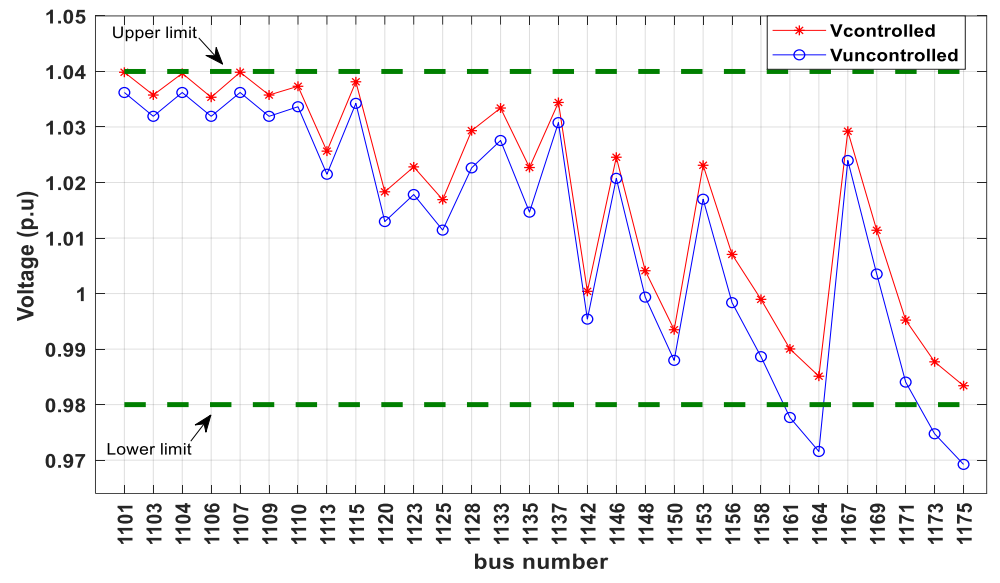

Fig.8. Voltage profile of the network for scenario 1.

To show the accuracy of the proposed sensitivities $\left|d Z_{L} / d Q\right|$ and $\left|d Z_{\text {eq }} / d Q\right|$, the contribution of each DG in the change of the impedances of the pilot bus, bus 1110, among some control actions is presented in Fig.9. It can be seen that the dominant change occurred by DG located near the pilot bus, DG at 1112. Contribution of other DGs in the change of the impedances of the pilot bus is almost equal. This is because the pilot bus has a weak coupling effect with other DGs located at other feeders. Although the system is successfully stable in this scenario, the proposed voltage control was able to improve the value of TLIM by $0.018 \%$.

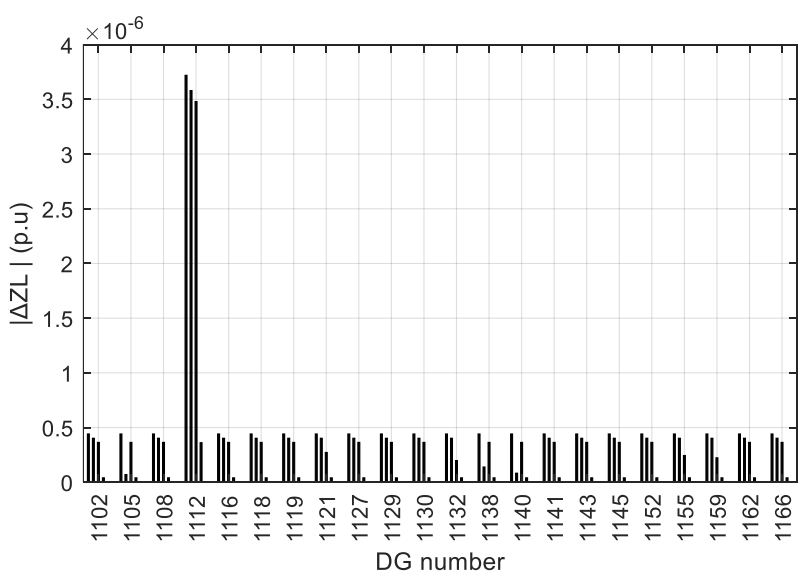

(a)

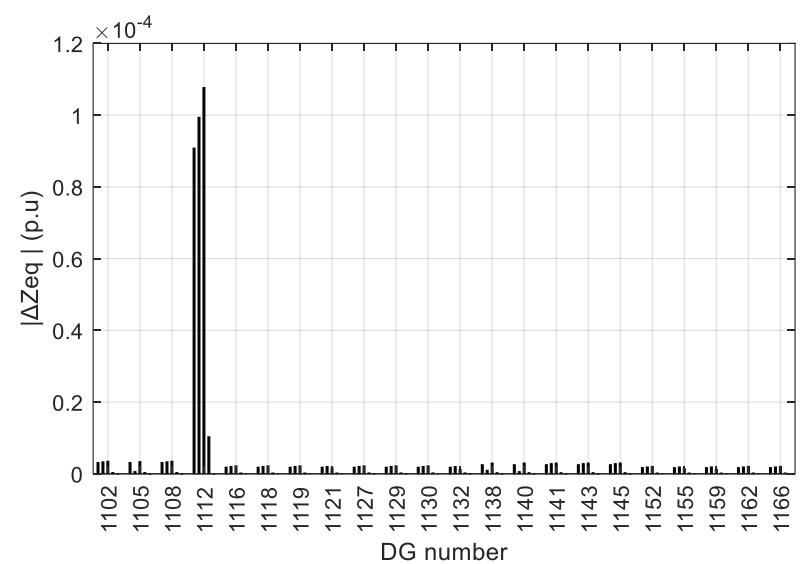

(b)

Fig.9. The change of the (a) load and (b) equivalent impedance of pilot bus caused by each DG among some control actions for scenario 1.

\subsubsection{DG Unavailability}

In this scenario, the system was operated so that all voltages are within normal voltages. It was assumed that the network met an outage of two DG units, located at the buses 1166 and 1162, at $\mathrm{t}=0 \mathrm{~s}$. This created low voltage conditions at some buses of feeder 4. Fig. 10 shows the voltages regulation and the changes in the reactive power starting at $\mathrm{t}=40 \mathrm{~s}$. It is clear that the rest of DG units can improve the voltages in case of the outage of some DG units.

To show the accuracy of the proposed sensitivities $\left|d Z_{L} / d Q\right|$ and $\left|d Z_{e q} / d Q\right|$, the contribution of each DG in the change of the impedances of pilot bus, bus 1175, among some control actions is presented in Fig.11. It can be seen that the dominant change occurred by the DGs located near pilot bus, bus 1175, and at the same feeder (located at buses $1159,1155 \& 1152$ ). The results also show the weak coupling effect of the pilot bus with other DGs located on other feeders. In this scenario, the proposed voltage control was able to improve the value of TLIM by $0.19 \%$. 


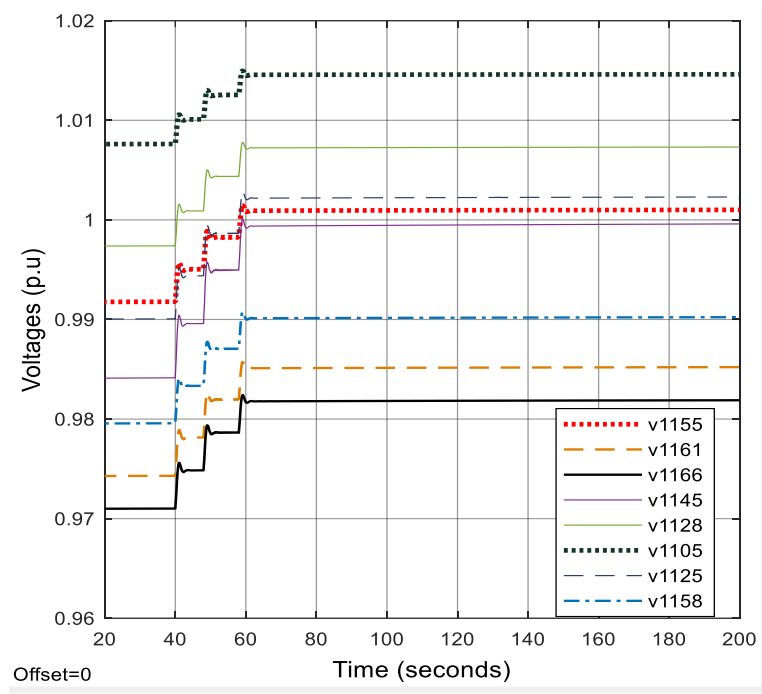

(a)

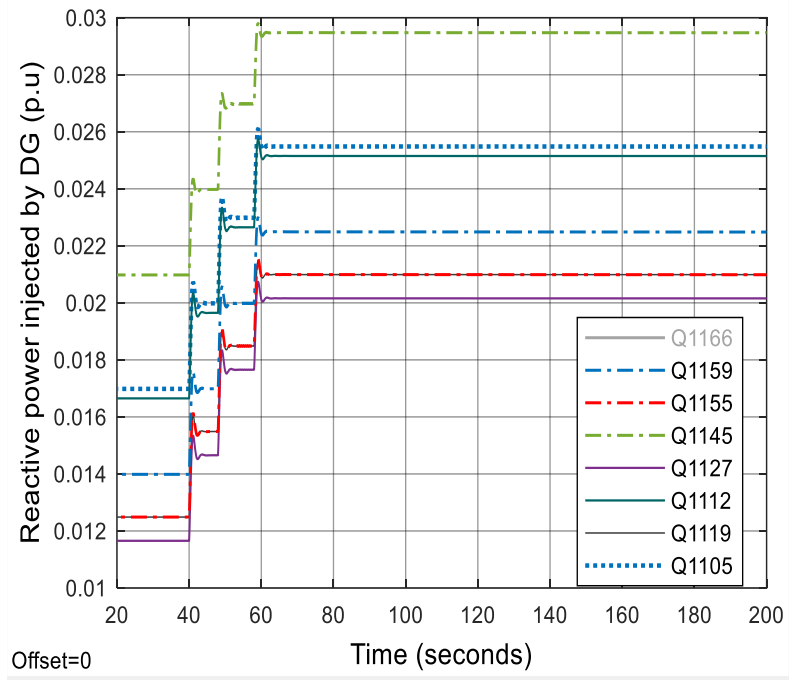

(b)

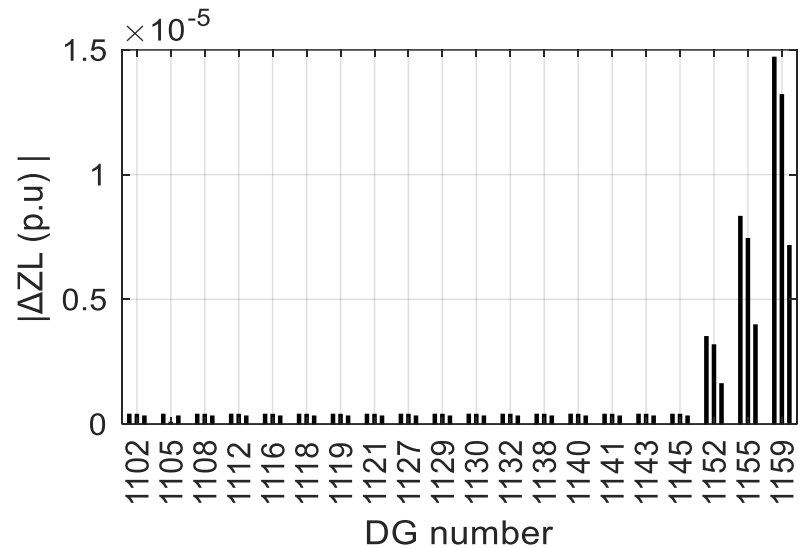

(a)

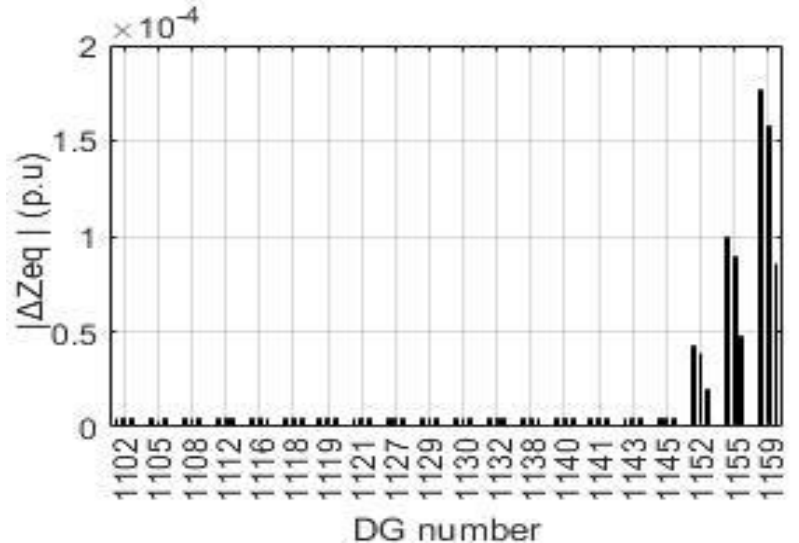

(b)

Fig.11. The change of the (a) load and (b) equivalent impedance of pilot bus caused by each DG among some control steps for scenario 2 .

\subsubsection{Over voltages scenario (Normal Scenario)}

To show the validity of the proposed voltage control during overvoltage conditions, the system was operated at low power consumptions and high power generated by DG units. Fig.12a shows the voltages regulation starting at $\mathrm{t}=20 \mathrm{~s}$ by adjusting the set point of DG units. Since there are some voltages exceeded the emergency upper limit, the controller used the reactive and active power outputs of DG units for voltage correction. Figs.12b and 12c show the reduction in active and reactive power outputs of some DG units, respectively. It is clear that as all the voltages are restored to nonemergency region, the controller only uses the reactive power outputs for voltage correction. This met at $t=40 \mathrm{~s}$. It is also shown that although most of the DG units absorbed reactive power, DG units located at the buses 1166 and 1145 were requested to still generate reactive power. This is because the voltages v1166 and v1145 would violate the lower limit of 0.98 p.u.

To show the accuracy of the proposed sensitivities $\left|d Z_{L} / d P\right|$ and $\left|d Z_{\text {eq }} / d P\right|$, the contribution of the change in active power outputs by each DG in the change of the impedances of pilot bus, bus 1110, is presented in Fig. 13. It can be seen that the dominant change occurred by DG located near pilot bus, DG at 1112, among the two control actions. 


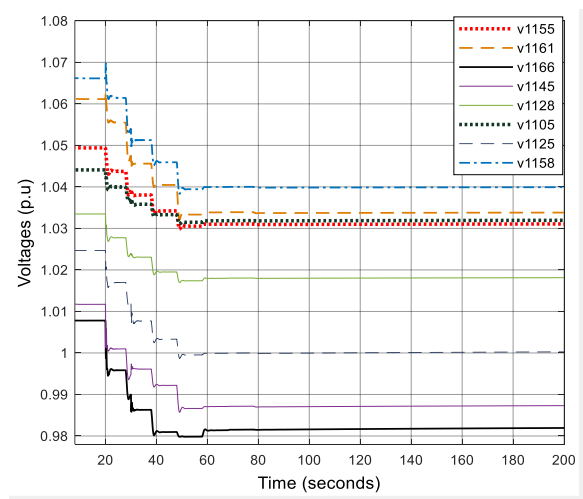

(a)

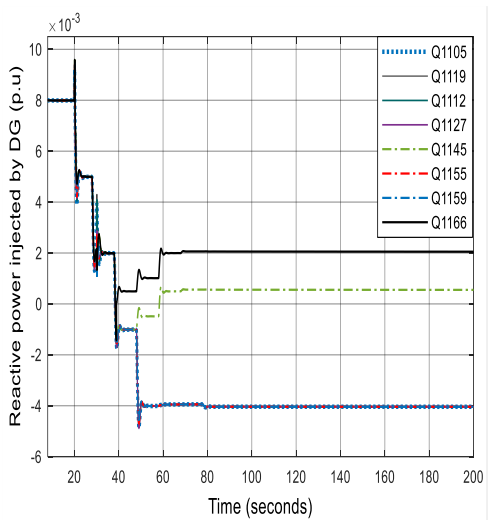

(b)

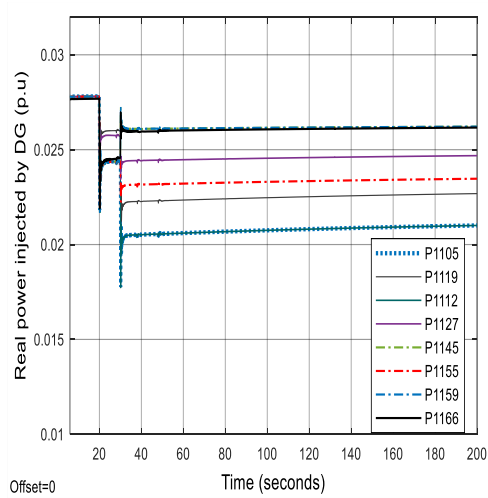

(c)

Fig. 12. (a) Bus voltage \& (b) Active and (c) Reactive power output of the DGs for scenario 3

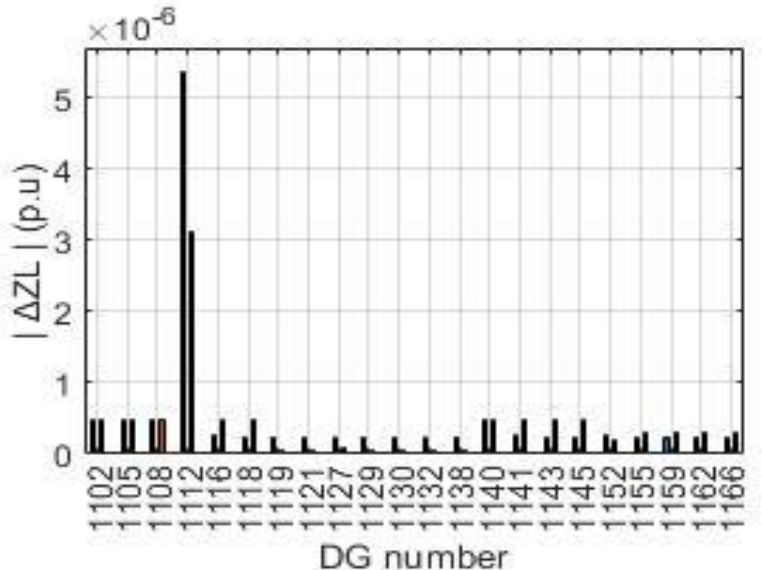

(a)

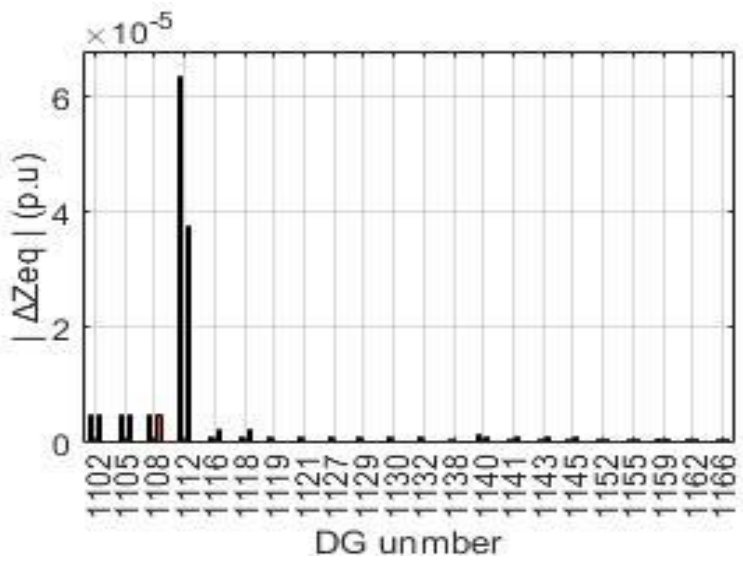

(b)

Fig. 13. The change of the (a) load and (b) equivalent impedance of pilot bus caused by the change in the real power output by each DG for Scenario 3 .

\subsubsection{Under voltages scenario (Emergency Scenario)}

To show the validity of the proposed voltage control during an extreme low voltage, it is assumed that the network is operated at high power consumptions and low power generated by DG units. Fig. 14a shows the voltages regulation starting at $\mathrm{t}=20 \mathrm{~s}$ by adjusting the set point of DG units. Fig. $14 \mathrm{~b}$ shows the gradual increase in reactive power outputs of some DG units. It is clear that as all the voltages are successfully brought back inside the normal limits by optimally dispatch the reactive power output by DG units. It can be seen from Fig.14.b that there is an overshoot in the control. This is due to the effect of slack values which used to relax some voltages.

To show the accuracy of the proposed sensitivities $\left|d Z_{L} / d Q\right|$ and $\left|d Z_{\text {eq }} / d Q\right|$, the contribution of the change in active power outputs by each DG in the change of the impedances of pilot bus, bus 1175, is presented in Fig. 15. It can be seen that the dominant change occurred by DG located near pilot bus, DG located among feeder 4.

In this scenario, the proposed voltage control was able to improve the value of TLIM by $1.92 \%$. 


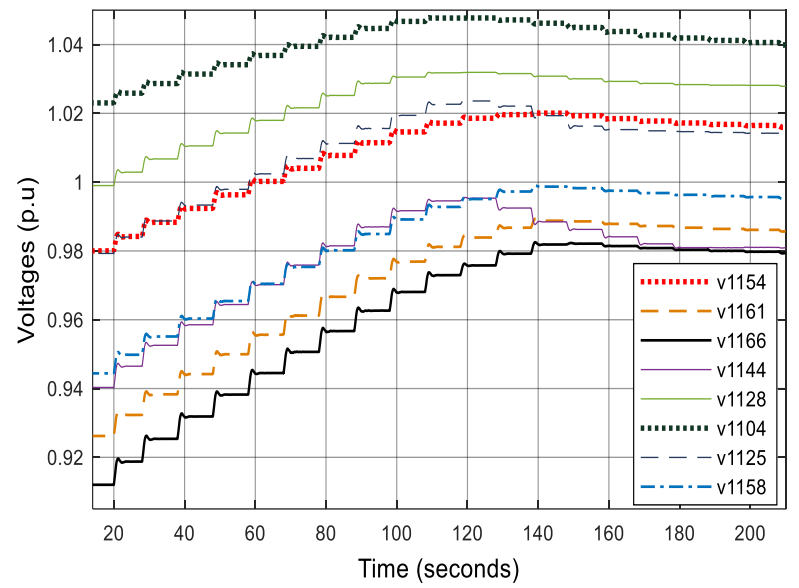

(a)

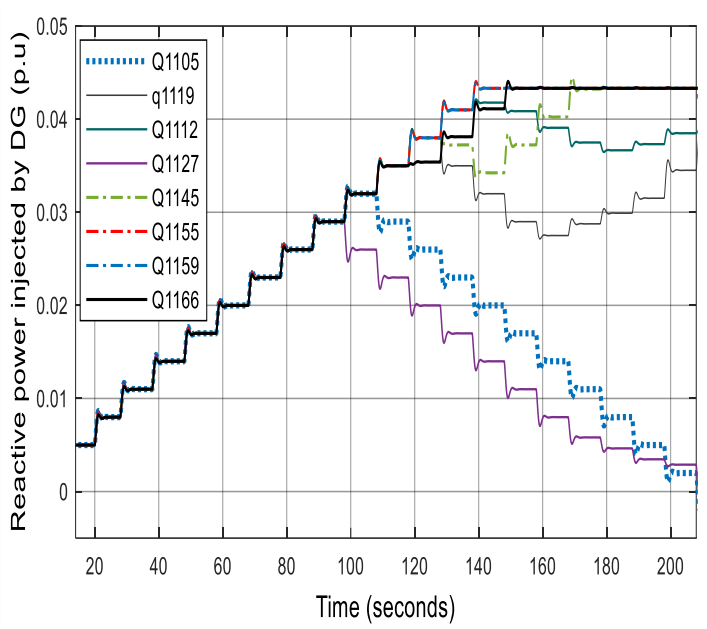

(b)

Fig. 14. (a) Bus voltage \&(b) Reactive power output of the DGs for scenario 4

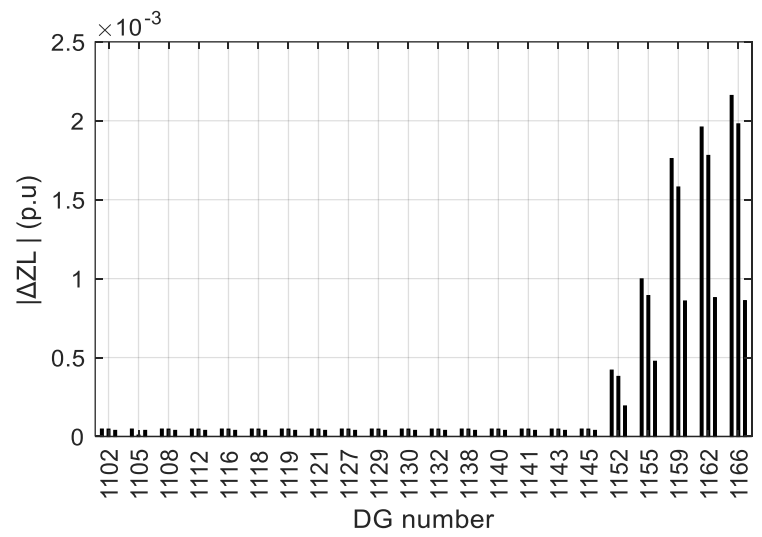

(a)

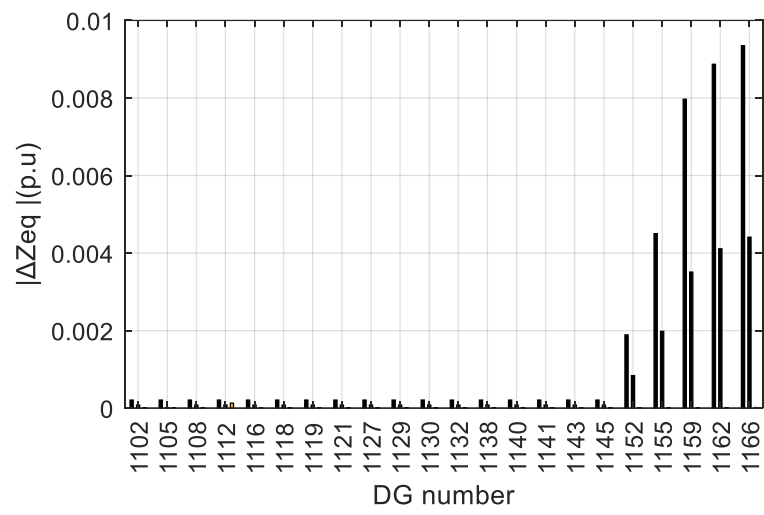

(b)

Fig. 15. The change of the (a) load and (b) equivalent impedance of pilot bus caused by each DG among some control steps for scenario 4.

\section{Conclusion}

This paper presents a new centralized voltage control method following a security purpose to optimally dispatch the control variables of active distribution grids. The method is based on the sensitivity of load and equivalent impedances to control variables. The method helps the distribution network operators to make a global voltage correction considering voltage stability issues inside distribution networks.

Simulation results show that the sensitivities of the load and equivalent impedances can accurately be used to quantitatively analyze the impact of change of power injections to the impedances. The results also show the accuracy and the validity of the proposed method in voltage control during different scenarios: normal and emergency operating conditions. It is also clear that the proposed method is applicable for real-time voltage control in the distribution network. This can be noticed from the frequent updated of the set points of control variables (every $10 \mathrm{~s}$ in this work).

Our future work is to develop appropriate PMU placement techniques to obtain the equivalent impedances with a full observability of the distribution systems. Moreover, application of the proposed method for a weak system and during contingency cases will be studied. 


\section{References}

[1] Y. M. Atwa and E. F. El-Saadany, Optimal allocation of ESS in distribution systems with a high penetration of wind energy, IEEE Trans. Power Syst. 25(4) (2010) 1815-1822.

[2] V. A. Evangelopoulos, P. S. Georgilakis, and N. D. Hatziargyriou, Optimal operation of smart distribution networks: A review of models, methods and future research, Electric Power Systems Research, 140 (2016) 95-106.

[3] O. Richardot, A. Viciu, Y. Bésanger, N. Hadjsaïd, and C. Kieny, Coordinated voltage control in distribution networks using distributed generation, In Transmission and Distribution Conference and Exhibition, IEEE PES, (2006) 1196-1201..

[4] M. Biserica, B. Berseneff, Y. Bésanger, C. Kiény, Upgraded coordinated voltage control for distribution systems, In PowerTech, 2011 IEEE Trondheim, (2011) 1-6.

[5] Q. Zhou and J. W. Bialek, Generation curtailment to manage voltage constraints in distribution networks, Generation Transm. Distribution, 1(3) (2007) 492-498.

[6] A. Borghetti, M. Bosetti, S. Grillo, S. Massucco, C. A. Nucci, M. Paolone, and F. Silvestro, Short-term scheduling and control of active distribution systems with high penetration of renewable resources, Syst. J., 4(3) (2010) 313-322.

[7] Oshiro, M., Tanaka, K., Senjyu, T., Toma, S., Yona, A., Saber, A. Y., \& Kim, C. H. Optimal voltage control in distribution systems using PV generators, International Journal of Electrical Power \& Energy Systems, 33(3)(2011) 485-492.

[8] Castro, J. R., Saad, M., Lefebvre, S., Asber, D., \& Lenoir, L., Optimal voltage control in distribution network in the presence of DGs, International Journal of Electrical Power \& Energy Systems, 78 (2016) 239-247.

[9] Dou, X., Duan, X., Hu, Q., Shen, L., \& Wu, Z. , A nonintrusive control strategy using voltage and reactive power for distribution systems based on PV and the nine-zone diagram, International Journal of Electrical Power \& Energy Systems, 105 (2019) 89-97.

[10] H.T. Yang, and J.T. Liao. , MF-APSO-based multiobjective optimization for PV system reactive power regulation, IEEE Transactions on Sustainable Energy, 6 (4) (2015) 1346-1355.

[11] A. Kulmala, S. Repo, and P. Järventausta, Coordinated voltage control in distribution networks including several distributed energy resources, IEEE Transactions on Smart Grid, 5 (4) (2014) 2010-2020.

[12] M. E. Baran and I. M. El-Markabi, A multiagent-based dispatching scheme for distributed generators for voltage support on distribution feeders, Trans. Power Syst., 22(1) (2007) 52-59.

[13] D. Jakus, J. Vasilj, and P. Sarajcev, Voltage control in MV distribution networks through coordinated control of tap changers and renewable energy sources, In PowerTech, 2015 IEEE Eindhoven, IEEE, (2015) 1-6.

[14] Degefa, M. Z., Lehtonen, M., Millar, R. J., Alahäivälä, A., \& Saarijärvi, E. , Optimal voltage control strategies for day-ahead active distribution network operation, Electric Power Systems Research, 127 (2015) 41-52.

[15] Li, C., Disfani, V. R., Pecenak, Z. K., Mohajeryami, S., \& Kleissl, J. , Optimal OLTC voltage control scheme to enable high solar penetrations, Electric Power Systems Research, 160 (2018) 318-326.

[16] Olival, P. C., Madureira, A. G., \& Matos, M. , Advanced voltage control for smart microgrids using distributed energy resources. Electric power systems research, 146 (2017) 132-140.

[17] Kryonidis, G. C., Demoulias, C. S., \& Papagiannis, G. K. , A new voltage control scheme for active medium-voltage (MV) networks, Electric Power Systems Research, 169 (2019) 53-64

[18] Meirinhos, J. L., Rua, D. E., Carvalho, L. M., \& Madureira, A. G. , Multi-temporal optimal power flow for voltage control in MV networks using distributed energy resources, Electric Power Systems Research, 146 (2017) 25-32.

[19] G. Valverde, and Th.Van Cutsem, Model predictive control of voltages in active distribution networks, IEEE Transactions on Smart Grid, 4(4) (2013) 2152-2161.

[20] Alamo, A. C. M., \& Alberto, L. F. C., A multi-step optimization approach for power flow with transient stability constraints, In 2015 IEEE Eindhoven PowerTech (2015, June),1-6.

[21] Guo, J., Tonguz, O., \& Hug, G., Impact of power system partitioning on the efficiency of distributed multi-step optimization. In 2017 IEEE Manchester PowerTech, (2017, June),1-6.

[22] P. A. Lof, T. Smed, G. Andersson, and D. J. Hill, Fast calculation of a voltage stability index, IEEE Trans. Power Systems, 7(1) (1992) 54-64.

[23] P. Kundur, Power System Stability and Control. McGraw-Hill Professional, 1994.

[24] M. H. Nazari, and M. Ilic, Dynamic modelling and control of distribution energy systems: comparison with transmission power systems, IET Generation, Transmission \& Distribution, 8(1) (2014) 26-34.

[25] J.H. Liu, and C.C. Chu, Long-term voltage instability detections of multiple fixed-speed induction generators in distribution networks using synchrophasors, IEEE Transactions on Smart Grid, 6(4) (2015) 2069-2079.

[26] X. Dou, S. Zhang, L. Chang, Z. Wu, W. Gu, M. Hu, \& X. Yuan., An improved CPF for static stability analysis of distribution systems with high DG penetration, International Journal of Electrical Power \& Energy Systems, 86 (2017) 177-188.

[27] K.Y, Liu, W. Sheng, L.Hu, Y. Liu, X. Meng, and D. Jia, Simplified probabilistic voltage stability evaluation considering variable renewable distributed generation in distribution systems, IET Generation, Transmission \& Distribution, 9(12) (2015) 1464-1473.

[28] H. M. Chou, and K. L. Butler-Purry, Investigation of Voltage Stability in Three-Phase Unbalanced Distribution Systems with DG using Modal Analysis Technique, In North American Power Symposium (NAPS), (2014) 1-6.

[29] F. Gubina, B. Strmcnik, A simple approach to voltage stability assessment in radial networks, IEEE Trans. Power Syst., 12(3) (1997) 11211128

[30] G.B. Jasmon, L.H.C.C. Lee, Distribution network reduction for voltage stability analysis and load flow calculations, Int. J. Electr. Power Energy Syst., 13 (1) (1991) 9-13.

[31] M.M. Hamada, , M.A.A. Wahab, N.G.A. Hemdan, Simple and efficient method for steady-state voltage stability assessment of radial distribution systems, Electr. Power Syst. Res., 80 (2010) 152-160.

[32] Wang, Yunfei, Iraj Rahimi Pordanjani, Weixing Li, Wilsun Xu, Tongwen Chen, Ebrahim Vaahedi, and Jim Gurney., Voltage stability monitoring based on the concept of coupled single-port circuit, IEEE Transactions on Power Systems, 26(4) (2011) 2154-2163.

[33] Alzaareer, K., \& Saad, M. , Real-Time Voltage Stability Monitoring in Smart Distribution Grids, International Conference on Renewable Energy and Power Engineering (REPE), IEEE, (2018) 13-17.

[34] Li, S., Tan, Y., Li, C., Cao, Y., \& Jiang, L. A Fast Sensitivity-Based Preventive Control Selection Method for Online Voltage Stability Assessment, IEEE Transactions on Power Systems, 33(4) (2018) 4189-4196.

[35] Matavalam, A. R. R., \& Ajjarapu, V., Sensitivity based thevenin index with systematic inclusion of reactive power limits, IEEE Transactions on Power Systems, 33(1) (2018) 932-942. 
[36] United Kingdom Generic Distribution Network (UKGDS). [Online]. Available: http://sedg.ac.uk 